\title{
Medición de la huella de carbono de la Universidad Nacional de Costa Rica para el periodo 2012-2014. Rumbo a la carbono neutralidad
}

Measurement of Carbon Footprint at National University of Costa Rica for 2012-2014. Heading for Carbon Neutrality

Fabián Chavarría-Solera

fabian.chavarria.solera@una.cr

Programa UNA-Campus Sostenible

Universidad Nacional

Heredia, Costa Rica

Óscar Mario Molina-León

oscar.molina.leon@una.cr

Universidad Nacional, Regencia Química

Heredia, Costa Rica

Rebeca Gamboa-Venegas

rebe.gamb07@gmail.com

Programa UNA-Campus Sostenible

Universidad Nacional

Heredia, Costa Rica

Jeison Rodríguez-Flores

jeison200916@hotmail.com

Programa UNA-Campus Sostenible

Universidad Nacional

Heredia, Costa Rica

Recibido-Received: 24/jun/2015/ Aceptado-Accepted: 8/set/2015/ Publicado-Published: 31/jul /2016

\begin{abstract}
Resumen
La cuantificación y medición de indicadores ambientales como la huella de carbono permite planear medidas encaminadas hacia una mejora de las condiciones que propician un impacto positivo en el entorno, disminuyendo o mitigando la cantidad de emisiones de dióxido de carbono y de otros gases que aceleran el efecto invernadero. La Universidad Nacional (UNA) pretende realizar la medición anual de este indicador, con el fin de poder implementar políticas y acciones que integren a la totalidad de la población universitaria encaminada hacia un buen desarrollo sustentable de la institución. En ese sentido, paralelo a la meta nacional planteada de C-Neutralidad, la UNA busca aportar en los esfuerzos por mitigar, minimizar o compensar el impacto ambiental, con un compromiso integral de su personal y estudiantes. El objetivo de este estudio es el de cuantificar y actualizar, en el periodo de 2012 a 2014, la huella de carbono de la UNA, para poder analizar y comparar su comportamiento en el tiempo, según los aspectos aplicables contemplados en el cálculo. Para el inventario de emisiones de gases de efecto invernadero (GEI) se aplicó la metodología avalada por el Instituto Meteorológico Nacional (IMN), utilizando los
\end{abstract}


factores oficializados, indicados en el Manual de factores de emisión de gases de efecto invernadero, en su cuarta edición del 2014. Como resultado, entre los años 2012 al 2014 se obtuvo un aumento del 22\% de la huella de carbono, pasando de 2.906 a 3.568 toneladas $\mathrm{CO}_{2}$ equivalente. Lo anterior debido a aumentos en varios aspectos, como la cantidad de personas en la institución, así como la contaminación emitida por los viajes aéreos. Con estos resultados se pretende identificar aumentos o disminuciones en las emisiones y establecer estrategias o medidas ambientales específicas para su reducción.

Palabras claves: Carbono, neutralidad, indicadores ambientales, dióxido de carbono, efecto invernadero, inventario.

\begin{abstract}
Quantifying and measuring environmental indicators such as carbon footprint allows to plan measures aimed towards an improvement of the conditions that are conducive to a positive impact on the environment, minimizing or mitigating emissions of carbon dioxide and other gases that accelerate the greenhouse effect. The National University of Costa Rica (UNA, in Spanish) seeks to make the annual measuring of this indicator, in order to implement policies and actions that integrate the entire university population routed to a good sustainable development of the institution. In that sense, parallel to the raised national goal of carbon neutrality, UNA seeks to contribute in efforts to mitigate, minimize or compensate the environmental impact, with a comprehensive commitment of its staff and its students. The objective of this study is to quantify and update carbon footprint at UNA for the 2012-2014 period, to be able to analyze and compare its behavior through time, according to the applicable aspects referred to in the calculation. For the inventory of greenhouse gases (GHG) the methodology applied was endorsed by the National Meteorological Institute (IMN, for its initials in Spanish), using timekeeping factors, specified in the Manual of Greenhouse Gases Emission Factors, in its fourth edition, 2014. As a result, between the years 2012 to 2014 a $22 \%$ increase of carbon footprint was obtained, going from the equivalent of 2,906 to 3,568 tonnes of $\mathrm{CO}_{2}$. The above due to increases in various aspects, as the number of people in the institution, as well as the pollution emitted by air travel. The intention of these results is to identify increases or decreases in emissions and establish strategies or specific environmental measures for their reduction.
\end{abstract}

Keywords: Carbon neutrality, environmental indicators, carbon dioxide, greenhouse effect, inventory.

En la actualidad, hablar de ambiente y de los impactos positivos o negativos que puede generar el ser humano sobre este es relativamente común. La mayoría de los países, organizaciones, empresas y otras tipos de instituciones tienen medidas para mitigar los impactos perjudiciales causados por la contaminación en general. Una de las principales fuentes de contaminación es la producida por los gases de efecto invernadero, los cuales, al liberarse a la atmósfera, crean una capa que impide la liberación de calor, lo que aumenta la temperatura del planeta. A este efecto se le conoce comúnmente como calentamiento global.

En el año 2006, Costa Rica adquirió el compromiso de llegar a ser neutral en carbono o "C-Neutral" para el año 2021. Debido a este compromiso, la meta esperada de la Universidad Nacional de Costa Rica es llegar a ser C-neutral, lo que implica buscar opciones para establecer una reducción de la cantidad de $\mathrm{CO}_{2}$ emitido y un balance o aumento entre la cantidad de $\mathrm{CO}_{2}$ fijado. "Todas las medidas a implementar conllevan un compromiso por parte de la comunidad universitaria, tanto de estudiantes como funcionarios, por apoyar y fortalecer la política ambiental existente, de cara al desafío planteado para el país dentro de su programa C-neutral para el año 2021" (Benavides, 2011, p. 138).

En ese sentido, la Universidad Nacional de Costa Rica (UNA) forma parte de estas instituciones que trabajan para disminuir el deterioro ambiental, mediante la implementación de un Programa de Gestión Ambiental Institucional (PGAI). Para ello se realiza la contabilización y cálculo de indicadores ambientales, los cuales son factores directos e indirectos necesarios para 
el cálculo de la huella de carbono como el consumo de energía eléctrica, combustibles fósiles de fuentes móviles y fijas, aguas residuales, fertilizantes, entre otros; que permiten realizar un inventario para tener una aproximación de la cantidad de gases de efecto invernadero (GEI) emitido por la institución. "El inventario es un retrato de un momento específico, por lo que debe ser actualizado una vez al año para poder identificar los principales cambios en las principales fuentes de emisión y realizar las acciones puntuales de reducción” (IMN, 2009, p.1 $)$.

El quehacer diario de las organizaciones está ligado al consumo de una serie de bienes y servicios (papel, energía, agua y otros), y a la respectiva generación de residuos (emisiones atmosféricas, residuos sólidos y aguas residuales) que van generando un impacto en el ambiente. "Lo que se busca en la institución por medio de los PGAI es la concientización de los impactos negativos generados asumiendo medidas ambiéntales de prevención, mitigación, restauración o compensación” (MINAET 2011, p. 51). Según la ISO 14067 (2013):

La Huella de Carbono es un parámetro utilizado para describir la cantidad de emisiones de gases de efecto invernadero (GEI) asociadas a una empresa, evento, actividad o al ciclo de vida de un producto/servicio para determinar su contribución al cambio climático. Se expresa en toneladas de $\mathrm{CO}_{2}$ equivalente (p. 14).

Con el cálculo de este tipo de indicadores se muestra el estado del desarrollo institucional en materia ambiental. Este sirve para la implementación de medidas para una adecuada gestión del cambio climático. Estos datos se proporcionan tanto netos como per cápita para mostrar el avance en relación con los resultados anteriores en la institución y con los resultados en general del país. "La Huella de Carbono, representa una medida para la contribución de las organizaciones a ser entidades socialmente responsables y un elemento más de concientización para la asunción entre los ciudadanos de prácticas más sostenibles" (Huella de Carbono, 2009, párraf. 2).

De acuerdo con Global Footprint Network (2015, párraf. 1):

El cambio del clima global es uno de los desafíos más grandes de la humanidad y uno de los indicadores más importantes ya que estamos en sobregiro ecológico. Dado que la huella de carbono es el 50 por ciento de la huella ecológica total de la humanidad, la reducción de nuestra huella de carbono es esencial en la solución del sobregiro ecológico.

Como consecuencia, la UNA apunta sus esfuerzos en materia ambiental a la búsqueda de la carbono neutralidad (C-Neutralidad), apoyada en la implementación de su Programa de Gestión Ambiental Institucional, pues considera estos esfuerzos importantes para la reducción de sus emisiones, a la vez que se minimizan sus impactos ambientales y se genera una mejor imagen ante la sociedad. Con la medición de la huella de carbono, la Universidad inicia el proceso de ser carbono neutral, lo que significa que las emisiones netas asociadas con las actividades de la institución sean iguales a cero. En búsqueda de este objetivo, la institución, integrada por todos los departamentos e instancias, así como por los actores principales -personal y estudiantes-, debe tomar medidas de reducción y compensación de las emisiones residuales.

Por lo anterior, el presente estudio muestra el inventario de las emisiones de gases de efecto invernadero que se ha venido realizando en la institución, según la metodología propuesta por el Instituto Meteorológico Nacional (IMN) para el cálculo de la huella de carbono. Se realiza la medición de la huella de carbono de los años 2012, 2013 y 2014 para, posteriormente, proceder 
a establecer estrategias o medidas de mitigación y prevención de los principales causantes de la emisión de gases de efecto invernadero; lo anterior se realiza en el marco de los PGAI, para lograr una mejora de la sostenibilidad ambiental y acercar aún más a la Universidad Nacional a la meta de ser carbono neutral.

\section{Metodología}

\section{Sitio de estudio}

El inventario de emisiones y posterior cálculo de las toneladas de dióxido de carbono equivalente se realizó en total para toda la institución, tomando en cuenta todos los campus universitarios, contemplando la totalidad del territorio donde la Universidad Nacional realiza sus actividades. Estas son:

Sedes: 1. Central (Campus Omar Dengo y Benjamín Núñez), 2. Brunca (Campus Coto y Pérez Zeledón), 3. Chorotega (Campus Liberia y Nicoya), Huetar Norte y Caribe (Sarapiquí). Cuatro Estaciones: (ECMAR -Estación Nacional de Ciencias Marino Costeras-EBM -Estación de Biología Marina-, Estación 28 millas, Estación Río Macho) y Finca Experimental Santa Lucía de la Escuela de Ciencias Agrarias.

\section{Inventario de emisiones de gases de efecto invernadero}

Para la medición de la huella de carbono de la Universidad Nacional se utilizaron los aspectos contemplados en la tabla 1 , los cuales incluyen las emisiones directas e indirectas y que, según el IMN, son los que mayormente contribuyen con las emisiones de GEI y deben ser contemplados en el cálculo. Las emisiones directas son aquellas que se producen en la combustión directa de combustibles fósiles y la emisión de $\mathrm{CO}_{2}$ por residuos; mientras que las emisiones indirectas se refieren a las emitidas por terceros en la elaboración de productos consumidos por la institución.

Tabla 1

Aspectos aplicables para el inventario de emisiones de $\mathrm{CO}_{2}$ equivalente y cálculo de la huella de carbono de la UNA

\begin{tabular}{l}
\hline \multicolumn{1}{c}{ Aspecto aplicable } \\
\hline Energía eléctrica \\
Combustible fósil \\
Gas licuado de petróleo LP en servicios de alimentación \\
Generación de residuos sólidos enviados a relleno sanitario \\
Aguas residuales \\
Viajes aéreos \\
Ganado \\
Cerdos \\
Otros animales (caballos, cabras, ovejas y aves de corral) \\
Fertilizantes
\end{tabular}

Nota: Elasboración propia de la investigación. 
Una vez que se cuenta con las cantidades totales anuales de cada uno de estos aspectos, se multiplican por su factor correspondiente de emisión y se obtiene la cantidad de $\mathrm{CO}_{2}$ emitido por cada aspecto. Estos factores son proporcionados por el Instituto Meteorológico Nacional (IMN), indicados en el Manual de Factores de emisión de gases de efecto invernadero (2014). La sumatoria de los totales de $\mathrm{CO}_{2}$ da como resultado la cantidad total de $\mathrm{CO}_{2}$ equivalente emitida por la institución en un periodo determinado.

Los aspectos de residuos a relleno y viajes aéreos fueron contemplados en el inventario de emisiones de GEI; sin embargo, hay que indicar que, a pesar de que no se tiene un control operativo ni financiero sobre estos, se contemplan como parte de una visión integral de las afectaciones, según las disposiciones de la Norma ISO 14064-1:2006 o bien, norma nacional INTE 12-01-06:2011 (en el caso de Costa Rica para obtener la marca País).

Los gases de efecto invernadero (GEI) cuantificados fueron: metano $\left(\mathrm{CH}_{4}\right)$, óxido nitroso $\left(\mathrm{N}_{2} \mathrm{O}\right)$ y dióxido de carbono $\left(\mathrm{CO}_{2}\right)$. El periodo de estudio comprende del 2012 al 2014. A continuación se presenta la metodología empleada según la fuente de emisión.

\section{Emisiones efecto del consumo de energía eléctrica}

Con la ayuda del Programa de Gestión Financiera (PGF) de la UNA se actualizó el listado de los números de contrato de medidores que existen en la Universidad, mediante el pago de los recibos del servicio de energía eléctrica por parte de este Programa. Con el registro de medidores actualizado se solicitaron los consumos mensuales a los diferentes proveedores del servicio: Empresa de Servicios Públicos de Heredia (ESPH), Instituto Costarricense de Electricidad (ICE), Compañía Nacional de Fuerza y Luz (CNFL) y Coopeguanacaste. Los datos mensuales suministrados se sumaron para obtener el dato anual en kilovatios hora (kWh) al año.

El cálculo de emisiones de $\mathrm{CO}_{2}$ mediante el consumo de energía eléctrica se realizó con la siguiente fórmula:

Ton $\mathrm{CO}_{2} \mathrm{e}=\mathrm{kWh} * \mathrm{~F}_{\mathrm{KWh}} / 1000 \mathrm{Kg} /$ ton

Dónde:

Ton $\mathrm{CO}_{2}$ e: Toneladas de dióxido de carbono equivalente.

kWh: Kilowatts hora de energía eléctrica consumida.

$\mathrm{F}_{\mathrm{KWh}}$ : Factor de emisión oficial del IMN, kilogramos de $\mathrm{CO}_{2}$ emitido por cada kilowatts hora consumido.

$1000 \mathrm{Kg} /$ ton: Factor de kilogramos a toneladas.

\section{Emisiones efecto del consumo de combustible fósil en la flotilla vehicular y plantas eléctricas}

La información sobre este consumo institucional se solicitó a la Sección de Transportes de la Universidad Nacional, la cual facilitó los datos de gasto mensual en colones de todas las instancias de la UNA que poseen vehículos y realizaron giras en los años contemplados en este estudio. Para obtener los litros (L) consumidos por mes, se dividió el dato del importe pagado por el precio del combustible al mes de la compra, según el registro histórico de precios por litro de combustible de la Refinería Costarricense de Petróleo (RECOPE). El consumo de combustible 
fósil de las fuentes móviles (flotilla vehicular) se estandarizó a diesel, ya que el 90\% es a base de este combustible y el consumo total de combustible de diesel supera el 95\% en la UNA (Sección de Transportes, UNA). Los datos resultantes segregados por mes para cada campus, recinto y estación de la UNA se sumaron para obtener el dato anual en litros.

El cálculo de emisiones de $\mathrm{CO}_{2}$ mediante el consumo de diesel se realizó con la siguiente fórmula:

Ton $\mathrm{CO}_{2} \mathrm{e}=\left[\left(\mathrm{L}^{\star} \mathrm{F}_{\mathrm{CO}_{2}}\right)+\left(\mathrm{L}^{\star} \mathrm{F}_{\mathrm{CH}^{4}} / 1000 \mathrm{~g} / \mathrm{Kg}^{\star} 21\right)+\left(\mathrm{L}^{\star} \mathrm{F}_{\mathrm{N} 2 \mathrm{O}} / 1000 \mathrm{~g} / \mathrm{Kg}^{\star} 310\right)\right] / 1000 \mathrm{Kg} /$ ton

Dónde:

Ton $\mathrm{CO}_{2}$ e: Toneladas de dióxido de carbono equivalente.

L: Litros de combustible diesel.

$\mathrm{F}_{\mathrm{CO} 2}$ : Factor de emisión oficial del IMN, kilogramos de $\mathrm{CO}_{2}$ emitido por cada litro de diesel.

$\mathrm{F}_{\mathrm{CH} 4}$ : Factor de emisión oficial del IMN, gramos de $\mathrm{CH}_{4}$ emitido por cada litro de diesel.

$\mathrm{F}_{\mathrm{N} 2 \mathrm{O}}$ : Factor de emisión oficial del IMN, gramos de $\mathrm{N}_{2} \mathrm{O}$ emitido por cada litro de diesel.

21: Potencial de calentamiento global del $\mathrm{CH}_{4}$ para conversión a $\mathrm{CO}_{2}$.

310: Potencial de calentamiento global del $\mathrm{N}_{2} \mathrm{O}$ para conversión a $\mathrm{CO}_{2}$.

$1000 \mathrm{Kg} /$ ton: Factor de kilogramos a toneladas.

\section{Emisiones efecto del gas licuado de petróleo (LP) de los servicios de alimentación}

El gas LP es comprado y consumido por los concesionarios de los servicios de alimentación de la Universidad. Fueron considerados dentro del cálculo debido a que están dentro de los campus universitarios y su consumo es normado por la Comisión del Sistema Institucional de Sodas y Afines de la Universidad Nacional (SISAUNA) en conjunto con el Programa UNACampus Sostenible. Los datos de libras (lb) del gas consumido fueron solicitados a cada dueño de la concesión de los servicios de alimentación que están dentro de todos los campus de la universidad. No se consideró el gas LP consumido en los laboratorios, debido a que este es muy poco y se considera despreciable para el cálculo.

El cálculo de emisiones de $\mathrm{CO}_{2}$ mediante el consumo de gas LP se realizó con la siguiente fórmula:

Ton $\mathrm{CO}_{2} \mathrm{e}=\mathrm{lb}^{*} 1 \mathrm{Kg} / 2205 \mathrm{lb}^{*} 1 \mathrm{~m}^{3} / 543,7 \mathrm{Kg} * 1000 \mathrm{~L} / 1 \mathrm{~m}^{3 *} \mathrm{~F}_{\mathrm{GLP}} / 1000 \mathrm{Kg} /$ ton

Dónde:

Ton $\mathrm{CO}_{2}$ e: Toneladas de dióxido de carbono equivalente.

lb: Libras de gas consumido.

Kg: Kilogramos.

2205: Factor de conversión de libras a kilogramos.

$\mathrm{m}^{3}$ : Metros cúbicos.

$543,7 \mathrm{Kg} / \mathrm{m}^{3}$ : Densidad del gas LP.

$1000 \mathrm{~L} / \mathrm{m}^{3}$ : Factor de metros cúbicos a litros.

$\mathrm{F}_{\mathrm{GLP}}$ : Factor de emisión oficial del IMN, kilogramos de $\mathrm{CO}_{2}$ emitido por cada litro de gas LP. $1000 \mathrm{Kg} /$ ton: Factor de kilogramos a toneladas. 
ISSN Electrónico: 2215-3470

DOI: http://dx.doi.org/10.15359/ru.30-2.4
UNICIENCIA Vol. 30, No. 2, pp. 47-62. Julio-diciembre, 2016. URL: www.revistas.una.ac.cr/uniciencia Email: revistauniciencia@una.cr

\section{Emisiones efecto de la generación de residuos sólidos enviados a relleno sanitario}

La cantidad de kilogramos de residuos sólidos no aprovechables enviados a relleno sanitario fue suministrada por el Programa de Desarrollo y Mantenimiento de la Infraestructura Institucional (PRODEMI). El cálculo de emisiones de $\mathrm{CO}_{2}$ mediante este aspecto se realizó con la siguiente fórmula:

Ton $\mathrm{CO}_{2} \mathrm{e}=\mathrm{N}_{\mathrm{Rs}}{ }^{*} \mathrm{~F}_{\mathrm{Rs}}{ }^{*} 21 / 1000 \mathrm{Kg} /$ ton

Dónde:

Ton $\mathrm{CO}_{2}$ e: Toneladas de dióxido de carbono equivalente.

$\mathrm{N}_{\mathrm{Rs}}$ : Cantidad de residuos sólidos en kilogramos enviados al relleno sanitario.

$\mathrm{F}_{\mathrm{Rs}}$ : Factor de emisión oficial del IMN, kilogramos de $\mathrm{CH}_{4}$ emitido por cada kilogramo de residuos sólidos.

21: Potencial de calentamiento global del $\mathrm{CH}_{4}$ para conversión a $\mathrm{CO}_{2}$. $1000 \mathrm{Kg} /$ ton: Factor de kilogramos a toneladas.

\section{Emisiones efecto de los viajes aéreos}

La Proveeduría Institucional y la Comisión de Junta de Becas de la Universidad Nacional suministraron la información de cantidad y países a los cuales realizaron viajes aéreos funcionarios y estudiantes de la universidad en los años correspondientes. Dentro de estos también se contemplan las becas dadas por el Departamento de Bienestar Estudiantil y la Federación de estudiantes FEUNA.

Con la herramienta Google Earth ${ }^{\oplus}$ se realizó un cálculo aproximado de los kilómetros recorridos en el viaje de ida y vuelta. Para efectos del cálculo aproximado se siguió el viaje regular de los vuelos en línea recta, según país de origen y destino. A cada uno de los viajes se les aplicó la siguiente fórmula:

Ton $\mathrm{CO}_{2} \mathrm{e}=\mathrm{N}_{\mathrm{v}}{ }^{*} \mathrm{Km}_{\mathrm{v}}{ }^{*} \mathrm{~F}_{\mathrm{KmV}} / 1000 \mathrm{Kg} /$ ton

\section{Dónde:}

Ton $\mathrm{CO}_{2}$ e: Toneladas de dióxido de carbono equivalente.

$\mathrm{N}_{\mathrm{v}}$ : Número de viajes según destino.

$\mathrm{Km}_{\mathrm{v}}$ : Kilómetros recorridos en el viaje de ida y vuelta según destino.

$\mathrm{F}_{\mathrm{KmV}}$ : Factor de kilogramos de $\mathrm{CO}_{2}$ emitidos por kilómetro viajado, según la calculadora de huella de carbono para el transporte aéreo de la Fundación Terra (2008).

$1000 \mathrm{Kg} /$ ton: Factor de kilogramos a toneladas.

\section{Emisiones efecto de las aguas residuales}

Para el cálculo se consideró el factor del IMN de descarga a ríos para aguas residuales domésticas. El dato de la cantidad de personas funcionarias y estudiantes fue suministrado por el Programa Desarrollo de Recurso Humanos y el Departamento de Registro respectivamente.

El cálculo de emisiones de $\mathrm{CO}_{2}$ debido a este aspecto se realizó mediante la siguiente fórmula: 
UNICIENCIA Vol. 30, No. 2, pp. 47-62. Julio-diciembre, 2016.

Ton $\mathrm{CO}_{2} \mathrm{e}=\mathrm{N}_{\mathrm{p}}{ }^{*} \mathrm{~F}_{\mathrm{Ar}}{ }^{*} 21 / 1000 \mathrm{Kg} /$ ton

Dónde:

Ton $\mathrm{CO}_{2}$ e: Toneladas de dióxido de carbono equivalente.

$\mathrm{N}_{\mathrm{p}}$ : Número de personas.

$\mathrm{F}_{\mathrm{Ar}}$ : Factor de emisión oficial del IMN, kilogramos de $\mathrm{CH}_{4}$ emitido por cada persona al año.

21: Potencial de calentamiento global del $\mathrm{CH}_{4}$ para conversión a $\mathrm{CO}_{2}$.

$1000 \mathrm{Kg} /$ ton: Factor de kilogramos a toneladas.

\section{Emisiones efecto de la utilización de fertilizantes}

Los datos de cantidad de sacos de fertilizantes utilizados en la institución durante los años correspondientes fueron suministrados por la Finca Santa Lucia y por la Sede Sarapiquí.

El cálculo de emisiones de $\mathrm{CO}_{2}$ debido a la utilización de fertilizantes se realizó mediante la siguiente fórmula:

Ton $\mathrm{CO}_{2} \mathrm{e}=\mathrm{N}_{\mathrm{SF}} * 45 \mathrm{Kg} * \% \mathrm{~N} / 100 * 1 \% / 100 * 1,5714 * 310 / 1000 \mathrm{Kg} /$ ton

Dónde:

Ton $\mathrm{CO}_{2}$ e: Toneladas de dióxido de carbono equivalente.

$\mathrm{N}_{\mathrm{SF}}$ : Cantidad de sacos de fertilizante.

$45 \mathrm{Kg}$ : Peso en kilogramos de un saco de fertilizante.

$\% \mathrm{~N}$ : Porcentaje de nitrógeno presente en el fertilizante.

$1 \%$ : Porcentaje de nitrógeno que se volatiliza.

1,5714: Transformación del nitrógeno $\mathrm{N}_{2}$ a óxido de dinitrógeno $\mathrm{N}_{2} \mathrm{O}$.

310: Potencial de calentamiento global del $\mathrm{N}_{2} \mathrm{O}$ para conversión a $\mathrm{CO}_{2}$.

$1000 \mathrm{Kg} /$ ton: Factor de kilogramos a toneladas.

\section{Emisiones efecto de la tenencia de animales}

El inventario de la cantidad de animales según el tipo fue suministrada por la finca Santa Lucía, la Escuela de Medicina Veterinaria y la Sede Sarapiquí. Dentro del cálculo se contempló el ganado, cerdos, caballos, ovejas, cabras y aves de corral que posee la institución.

Ganado proceso digestivo: la cantidad total de ganado fue clasificado por tipo: terneros, hembras en crecimiento, machos en crecimiento, hembra adulta y macho adulto. De la clasificación, según el propósito, se utilizó solo leche, debido a que estos animales se utilizan con fines académicos e investigativos y no para la producción de carne.

Ganado manejo de estiércol: para la cantidad total de ganado se sumó el total, sin importar el tipo de ganado.

El cálculo de emisiones de $\mathrm{CO}_{2}$ debido a este aspecto se realizó mediante la siguiente fórmula:

Ton $\mathrm{CO}_{2} \mathrm{e}=\left[\left(\mathrm{N}_{\mathrm{GC}}{ }^{*} \mathrm{~F}_{\mathrm{G} \text { proc dig }}\right)+\left(\mathrm{N}_{\mathrm{GT}} * \mathrm{~F}_{\mathrm{G} \text { man est }}\right)\right] * 21 / 1000$ ton $/ \mathrm{Kg}$ 
ISSN Electrónico: 2215-3470

DOI: http://dx.doi.org/10.15359/ru.30-2.4
UNICIENCIA Vol. 30, No. 2, pp. 47-62. Julio-diciembre, 2016.

URL: www.revistas.una.ac.cr/uniciencia Email: revistauniciencia@una.cr

Dónde:

Ton $\mathrm{CO}_{2}$ e: Toneladas de dióxido de carbono equivalente.

$\mathrm{N}_{\mathrm{GC}}$ : Cantidad de ganado según la clasificación por tipo.

$\mathrm{F}_{\text {Gprocdig }}$ : Factor de emisión oficial del IMN, kilogramos de $\mathrm{CH}_{4}$ emitido por el ganado en el proceso digestivo según tipo y propósito.

$\mathrm{N}_{\mathrm{GT}}$ : Cantidad de ganado total.

$\mathrm{F}_{\mathrm{G} \text { manest }}:$ Factor de emisión oficial del IMN, kilogramos de $\mathrm{CH}_{4}$ emitido por el total de ganado en el manejo de estiércol.

21: Potencial de calentamiento global del $\mathrm{CH}_{4}$ para conversión a $\mathrm{CO}_{2}$.

1000 ton/Kg: Factor de kilogramos a toneladas.

Cerdos, caballos, ovejas y cabras proceso digestivo y manejo de estiércol: A la cantidad total del animal correspondiente que pertenecía a la Universidad para los años en estudio se le aplicó la siguiente fórmula para calcular la emisión de $\mathrm{CO}_{2}$ debido a este aspecto:

Ton $\mathrm{CO}_{2} \mathrm{e}=\left[\left(\mathrm{N}_{\mathrm{AT}}{ }^{*} \mathrm{~F}_{\text {Adproc dig }}\right)+\left(\mathrm{N}_{\mathrm{AT}}{ }^{*} \mathrm{~F}_{\text {Adman est }}\right)\right] * 21 / 1000$ ton $/ \mathrm{Kg}$

Dónde:

Ton $\mathrm{CO}_{2}$ e: Toneladas de dióxido de carbono equivalente.

$\mathrm{N}_{\mathrm{AT}}$ : Cantidad total del animal correspondiente (cerdos, caballos ovejas y cabras).

$\mathrm{F}_{\text {Cdprocdig }}$ : Factor de emisión oficial del IMN, kilogramos de $\mathrm{CH}_{4}$ emitido por el animal correspondiente en el proceso digestivo.

$\mathrm{F}_{\text {Cdmanest }}$ : Factor de emisión oficial del IMN, kilogramos de $\mathrm{CH}_{4}$ emitido por el animal correspondiente en el manejo de estiércol.

21: Potencial de calentamiento global del $\mathrm{CH}_{4}$ para conversión a $\mathrm{CO}_{2}$.

1000 ton/Kg: Factor de kilogramos a toneladas.

Aves de corral manejo de estiércol: La cantidad total de aves de corral que pertenecían a la universidad para los años correspondientes se les aplicó la siguiente fórmula para calcular la emisión de $\mathrm{CO}_{2}$ debido a este aspecto:

Ton $\mathrm{CO}_{2} \mathrm{e}=\mathrm{N}_{\mathrm{Ac}}{ }^{*} \mathrm{~F}_{\text {Ac man est }}{ }^{*} 21 / 1000$ ton $/ \mathrm{Kg}$

Dónde:

Ton $\mathrm{CO}_{2} \mathrm{e}$ : Toneladas de dióxido de carbono equivalente.

$\mathrm{N}_{\mathrm{Ac}}$ : Cantidad de aves de corral total.

$\mathrm{F}_{\text {Avmanest }}$ : Factor de emisión oficial del IMN, kilogramos de $\mathrm{CH}_{4}$ emitido por el total de aves de corral en el manejo de estiércol.

21: Potencial de calentamiento global del $\mathrm{CH}_{4}$ para conversión a $\mathrm{CO}_{2}$. 1000 ton/Kg: Factor de kilogramos a toneladas. 
UNICIENCIA Vol. 30, No. 2, pp. 47-62. Julio-diciembre, 2016.

Email: revistauniciencia@una.cr

\section{Resultados y discusión}

El comparativo de las cantidades de toneladas de $\mathrm{CO}_{2}$ anuales emitidas por la Universidad Nacional de Costa Rica, según los diferentes aspectos considerados en el inventario de la huella de carbono para los tres años de estudio, se muestran la figura 1.

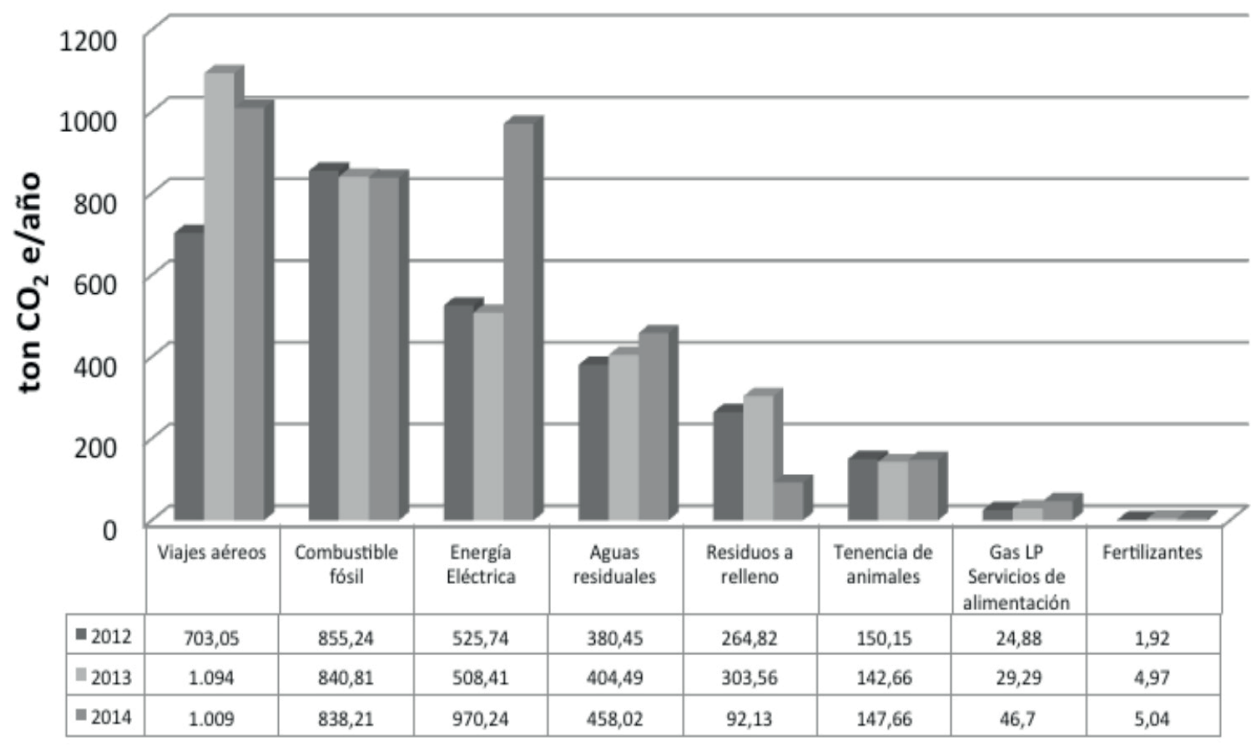

Figura 1. Gráfico comparativo de las toneladas de $\mathrm{CO}_{2}$ emitidas por la Universidad Nacional de Costa Rica para los años 2012, 2013 y 2014, según los diferentes aspectos considerados en el estudio (Elaboración propia de la investigación).

Viajes aéreos: Este aspecto es el de mayor emisión de $\mathrm{CO}_{2}$ equivalente registrado en la Universidad Nacional; se puede ver una variabilidad considerable año tras año, ya que el personal que viaja lo hace a zonas muy diferentes a nivel global, así como la cantidad de funcionarios y funcionarias que viajan anualmente, lo cual ocasiona que las emisiones no presenten una tendencia predictiva. Sin embargo, en los dos últimos años de estudios se ha emitido un nivel del orden de las 1000 ton $\mathrm{CO}_{2}$ equivalente.

Combustible fósil: El consumo en este aspecto muestra una leve disminución de emisión, la cual se puede achacar a la sistematización e implementación de un sistema informático con el que se mejoró la eficiencia para la solicitud de giras utilizadas para la investigación y academia, la eficiencia de giras cortas, además de sensibilización y capacitaciones sobre manejo eficiente para mejorar las buenas prácticas de conducción y el mantenimiento correctivo y preventivo de los vehículos institucionales. Por otra parte, una de las nuevas medidas que están siendo implementadas es la adquisición de un sofisticado sistema de GPS en algunos vehículos de la flotilla institucional, lo que permitirá una adecuada gestión del consumo y ahorro del combustible. Todas estas medidas realizadas son para asegurar la utilización más eficiente de este insumo, en beneficio de un aprovisionamiento de demandas futuras. 
Energía eléctrica: Como se mencionó anteriormente, dicho aspecto tuvo un incremento considerable del 2013 al 2014, del 90,84\%. Dicho aumento se debió a una serie de factores que vinieron a generar un mayor consumo como, por ejemplo, el constante incremento en la población universitaria, la cual aumentó alrededor de un $20 \%$. En esta población se contempla a funcionarios, estudiantes regulares y estudiantes de cursos libres de todas las instancias de la UNA (tabla 2). Otro aspecto importante a mencionar es el aumento en los medidores por concepto de alquileres para centros de trabajo de la institución. Por último, existe un aumento en la infraestructura de la UNA con la construcción de nuevos edificios y la activación de más medidores de energía eléctrica. Durante el año 2012 estuvo en construcción nueva infraestructura, tal es el caso del edificio de Informática en el campus Benjamín Núñez, el cual ya está en funcionamiento y representa un $6 \%$ del total de kWh al año de la institución.

Aguas residuales: El aumento en este aspecto de las emisiones anuales de $\mathrm{CO}_{2}$ equivalente se debe, principalmente, al incremento en la población universitaria (tabla 2) en donde se ha venido incrementando la matrícula de estudiantes de la universidad, ya que la relación es proporcional, a mayor población se da una mayor generación de aguas residuales, según la metodología establecida por el Instituto Meteorológico Nacional.

Residuos a relleno: Si bien del año 2012 al 2013 hubo un incremento en las emisiones generadas por los residuos que terminaban en el relleno, se dio una disminución bastante considerable (69,65\%) del 2013 al 2014. Esta, probablemente, se debió a una constante capacitación a la población estudiantil, lo cual busca generar una concientización para que los residuos sólidos aprovechables no terminen en un relleno sanitario y, por el contrario, sean valorizados mediante una gestión integral, separándolos por tipos para ser enviados a reciclar con empresas gestoras autorizadas para su tratamiento. Además se implementaron, con afiches y calcomanías, los rótulos informativos sobre el tipo de material que debe ir en cada contenedor de separación. Estas medidas implementadas han hecho que se separen alrededor de 100 toneladas de residuos aprovechables para el reciclaje.

Ganado, cerdos, otros animales y fertilizantes: La cantidad de animales de granja de la institución y el fertilizante aplicado en las instalaciones de la Universidad Nacional, durante los tres años, no ha cambiado sustancialmente. Esto ocasiona que no existan variaciones significativas durante el periodo de estudio, en estos cuatro aspectos. Además, los cuatro aspectos representan solamente en promedio durante los tres años un 4,65\% de la cantidad de $\mathrm{CO}_{2}$ equivalente emitido por la universidad.

Gas LP servicios de alimentación: En este aspecto se nota un incremento anual, donde el más considerable se da del año 2013 al 2014. En estos años se incorporaron nuevos servicios de alimentación, además del incremento en la cantidad de usuarios del servicio, debido al aumento de la población universitaria en un $15,41 \%$, lo que pudo ocasionar el incremento en consumo de gas LP y, por ende, un aumento en la emisión de $\mathrm{CO}_{2}$ equivalente.

Los resultados obtenidos en la huella de carbono, según el inventario de gases de efecto invernadero (GEI) realizado para las emisiones de estos aspectos consideradas en la UNA en el periodo 2012-2014, se muestran en la tabla 2 y en la figura 2.

Como se puede observar, entre el año 2012 y 2013 se aumentó la huella de carbono pasando de 2906 a 3328 toneladas de $\mathrm{CO}_{2}$ equivalente, en términos porcentuales el incremento es de $14,52 \%$, debido principalmente a un aumento de los viajes aéreos, residuos a relleno sanitario y utilización de fertilizantes, en ese orden. 
UNICIENCIA Vol. 30, No. 2, pp. 47-62. Julio-diciembre, 2016.

URL: www.revistas.una.ac.cr/uniciencia

Email: revistauniciencia@una.cr
ISSN Electrónico: 2215-3470

DOI: http://dx.doi.org/10.15359/ru.30-2.4

Tabla 2

Resultados del cálculo de la huella de carbono para los años 2012, 2013 y 2014

\begin{tabular}{llllll}
\hline \multirow{2}{*}{ Aspecto aplicable } & \multicolumn{3}{c}{ Emisiones anuales $\left(\right.$ ton $\mathbf{C O}_{2}$ e) } & \multirow{2}{*}{ Total ton } & \multirow{2}{*}{ Emisión\% } \\
\cline { 2 - 5 } & $\mathbf{2 0 1 2}$ & $\mathbf{2 0 1 3}$ & $\mathbf{2 0 1 4}$ & $\mathbf{C O 2}$ & \\
\hline Viajes aéreos & 703,05 & $1.093,70$ & $1.008,83$ & $2.805,58$ & 29 \\
Combustible fósil & 855,24 & 840,81 & 838,21 & $2.534,26$ & 26 \\
Energía eléctrica & 525,74 & 508,41 & 970,24 & $2.004,39$ & 20 \\
Aguas residuales & 380,45 & 404,49 & 458,02 & $1.242,96$ & 13 \\
Residuos a relleno sanitario & 264,82 & 303,56 & 92,13 & 660,51 & 7 \\
Ganado & 122,55 & 120,96 & 126,29 & 369,8 & 4 \\
Gas LP servicios de alimentación & 24,88 & 29,29 & 46,7 & 100,87 & 1 \\
Otros animales (caballos, cabras, ovejas aves de & 27,26 & 21,15 & 20,82 & 69,23 & 1 \\
corral) & 1,92 & 4,97 & 5,04 & 11,93 & 0,12 \\
Fertilizantes & 0,34 & 0,55 & 0,55 & 1,44 & 0,01 \\
Cerdos & $\mathbf{2 . 9 0 6 , 2 5}$ & $\mathbf{3 . 3 2 7 , 9 0}$ & $\mathbf{3 . 5 6 8 , 0 5}$ & $\mathbf{9 . 8 0 2 , 2 0}$ & $\mathbf{1 0 0}$ \\
\hline Total & 20.683 & 21.574 & 24.898 & - & - \\
\hline Población (estudiantes + funcionarios) & $\mathbf{0 , 1 4 0}$ & $\mathbf{0 , 1 5 4}$ & $\mathbf{0 , 1 4 3}$ & - & - \\
\hline Total per cápita & & & & & \\
\hline
\end{tabular}

Nota: Elaboración propia de la investigación.

Entre los años 2013 y 2014 se obtuvo un aumento del 7,21\%, pasando de 3328 a 3568 toneladas $\mathrm{CO}_{2}$ equivalente; lo anterior debido a aumentos en varios aspectos como la cantidad de personas, además del aumento del factor de emisión de gases para efecto invernadero para el consumo eléctrico, aunado a esto una mayor cantidad en este consumo; por otro lado, el consumo de gas LP para los servicios de alimentación creció en un 59\% aproximadamente entre estos dos años.

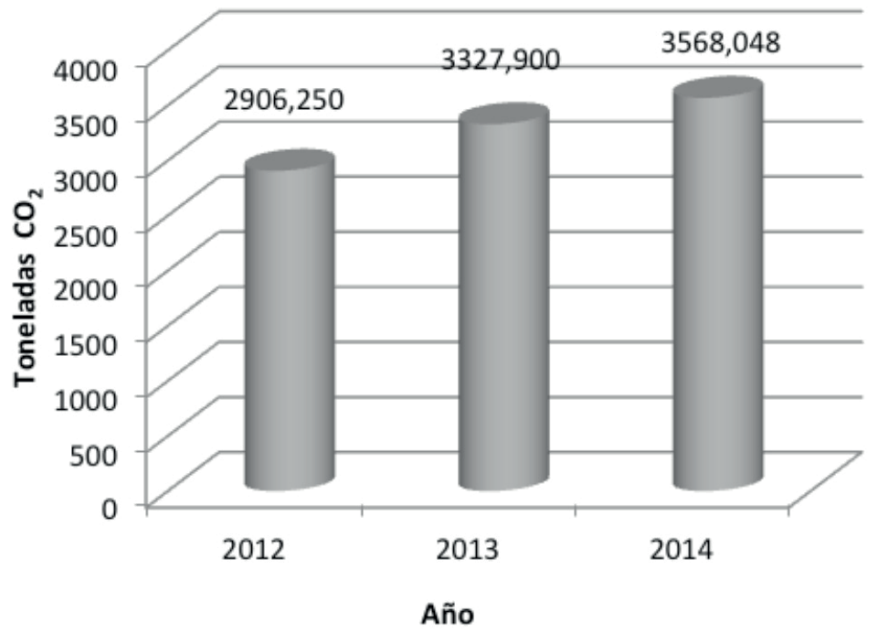

Figura 2. Gráfico comparativo de las toneladas de $\mathrm{CO}_{2}$ emitidos por la Universidad Nacional de Costa Rica para los años 2012, 2013 y 2014. Elaboración propia de la investigación. 
Según la tabla 2 y la figura 1, podemos observar que el aspecto que presentó una mayor emisión de $\mathrm{CO}_{2}$ equivalente, son los viajes aéreos representando un $29 \%$ total del período de estudio, en segundo lugar se encuentra el consumo de combustible en la flotilla vehicular con un $26 \%$ y en tercer lugar el consumo eléctrico institucional con un $20 \%$.

En el año 2012 estos tres componentes representaban el 71,71\% del total de emisiones anuales; en el 2013 estos representaban el 73,41\% y para el 2014 un 78,96\%; se puede evidenciar este incremento en emisiones generadas debido al consumo por concepto de "energía eléctrica" que se dio del año 2013 al 2014, dicho incremento se pude deber a una serie de construcciones que se desarrollaron en la universidad en ese periodo, además al cambio en el factor de emisión proporcionado por el Instituto Meteorológico Nacional que pasó en el 2013 de 0,0771 Kg de $\mathrm{CO}_{2}$ e/ KWh a 0,1300 Kg de $\mathrm{CO}_{2}$ e/ KWh en el año 2014 (anexos, tabla 3).

Aunque Costa Rica tiene una matriz energética asentada en fuentes limpias con un mayor porcentaje por generación hidroeléctrica, geotérmica y eólica, la generación basada en el uso de biomasa (biogás, residuos vegetales, bagazo y otros) así como la generación térmica basada en el uso de combustible fósil (búnker y diesel) aumenta o disminuye porcentualmente cada año (MINAE y IMN, 2014), afectando el factor de cálculo de $\mathrm{Kg}$ de $\mathrm{CO}_{2}$ e/ KWh oficializado por el IMN. En ese sentido, según el IMN, en el 2013 se utilizó más la generación térmica por biomasa aumentando considerablemente el factor.

Por su parte, en la figura 3 se observa la huella de carbono de la institución a nivel per cápita. A pesar de que cada año se ha aumentado la cantidad de toneladas de $\mathrm{CO}_{2}$ equivalente, a nivel poblacional se ha mantenido relativamente estable en cuanto a aumento o disminución. Para los años 2012 y 2013 la huella de carbono per cápita presentó un aumento mínimo, pasando de 0,140 a 0,154 toneladas $\mathrm{CO}_{2}$ /persona respectivamente. Posteriormente, para el año 2014, aunque la población creció en un $15,41 \%$ pasando de 21.574 a 24.898 personas, se dio una disminución de la huella de carbono per cápita con un valor de 0,143 ton $\mathrm{CO}_{2} /$ persona. Esta es una huella de carbono per cápita mínima en comparación con la reportada por el Banco Mundial (2015) para Costa Rica, que es de 2,55 toneladas al año por persona.

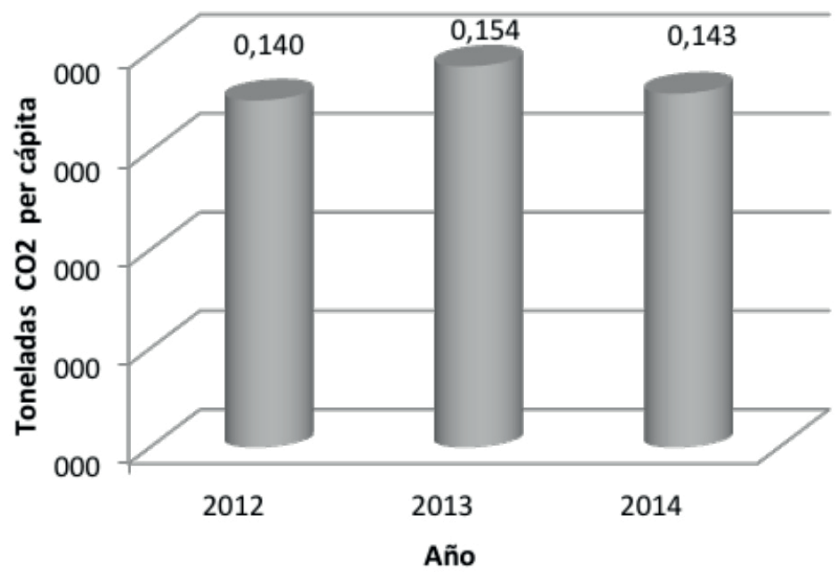

Figura 3. Gráfico comparativo de las toneladas de $\mathrm{CO}_{2}$ per cápita emitidas por la Universidad Nacional de Costa Rica para los años 2012, 2013 y 2014. Elaboración propia de la investigación. 
UNICIENCIA Vol. 30, No. 2, pp. 47-62. Julio-diciembre, 2016.

ISSN Electrónico: 2215-3470

URL: www.revistas.una.ac.cr/uniciencia

Email: revistauniciencia@una.cr

\section{Conclusiones}

En conclusión, la UNA emitió un total de 9802 ton/año $\mathrm{CO}_{2}$ equivalente durante el periodo 2012-2014. Las fuentes de emisión evaluadas que presentaron una mayor cantidad de $\mathrm{CO}_{2}$ equivalente emitido fueron, en primer lugar, los viajes aéreos (29\%); en segundo lugar, el consumo de combustible en la flotilla vehicular (26\%) y, en tercer lugar, el consumo de energía eléctrica institucional (20\%), seguido de las aguas residuales (13\%) y, por último, los residuos a relleno sanitario (7\%). Con estos cinco aspectos se tiene un $95 \%$ de las emisiones para los tres años de estudio.

El incremento general de la huella de carbono fue de 22\% (2012-2014). Entre los principales aspectos relacionados con este crecimiento se destaca el incremento de la población institucional en un 20,4\%, aspecto relacionado directamente con consumo de gas LP y energía eléctrica, aguas residuales y residuos a relleno; además de un aumento en las emisiones GEI indirectas como el consumo de gas LP, el cual es utilizado en los servicios de alimentación de la Universidad Nacional, que aumentó en un $88 \%$, sumado a un $43,5 \%$ debido a viajes aéreos considerados no controlables y a un aumento del $84,5 \%$ en la cantidad de $\mathrm{CO}_{2}$ emitido por el consumo eléctrico que depende del cambio en matriz de generación del país y, por lo tanto, en el cambio en el factor para el cálculo.

En cuanto al cálculo de la huella de carbono a nivel per cápita se pudo determinar que no ha habido aumentos significativos, manteniéndose relativamente constante en el periodo de estudio. Sin embargo, el mínimo aumento en el 2013 se debió a que solo se aumentó en un 4 \% la cantidad poblacional para ese año, en comparación con el $15 \%$ de aumento entre el año 2013 y 2014. En ese sentido, se puede indicar que las actividades en pro de la sostenibilidad ambiental realizadas por el Programa de Gestión Ambiental Institucional han ayudado al cumplimiento de sus objetivos de corto plazo en cuanto a la implementación de buenas prácticas ambientales, la sensibilización de la población universitaria para hacer un uso más eficiente de los recursos naturales e institucionales, así como la disminución en la cantidad de los residuos generados.

Para poder controlar mejor las emisiones GEI por concepto del consumo del gas LP se está elaborando un manual denominado Buenas prácticas ambientales en los servicios de alimentación de la Universidad Nacional, instructivo ambiental para normar y controlar este y otros aspectos como el manejo de residuos orgánicos, limpieza de trampas de grasa, manejo de residuos de aceite, utilización de composteras, utilización de productos de limpieza amigables con el ambiente y evaluaciones ambientales. Este será presentado y avalado en el Sistema Institucional de Sodas y Afines (SISAUNA), sistema que rige los servicios de alimentación, y posteriormente será entregado a cada ente concesionario a cargo del servicio.

Poco a poco la ejecución de proyectos e iniciativas ha permitido la incorporación de elementos de innovación por medio del uso de nuevas herramientas tecnológicas que permitirán mejorar la gestión del cambio climático y promover una mayor conciencia ambiental. Por su parte, con estos datos obtenidos, se espera dar el siguiente paso en cuanto a establecer y calcular las reducciones y compensaciones para obtener un total de cero emisiones y lograr que la Universidad Nacional de Costa Rica adquiera, en un corto plazo, la carbono neutralidad. Para esto, es necesario enfocarse en el engranaje y trabajo conjunto, mediante una comisión de varias instancias de la UNA, que realizan medidas ambientales 
ISSN Electrónico: 2215-3470

DOI: http://dx.doi.org/10.15359/ru.30-2.4
UNICIENCIA Vol. 30, No. 2, pp. 47-62. Julio-diciembre, 2016.

URL: www.revistas.una.ac.cr/uniciencia Email: revistauniciencia@una.cr

para la reducción de GEI debido a su quehacer diario, así como aquellas instancias que trabajan en proyectos ambientales considerados como compensación a esta emisiones y que, además, poseen información y datos importantes para que se pueda cumplir con este objetivo.

\section{Referencias}

Banco Mundial. (2015). Emisiones de todos los gases de efecto invernadero (toneladas métricas per cápita). Indicadores del desarrollo mundial. Centro de Análisis de Información sobre Dióxido de Carbono, División de Ciencias Ambientales del Laboratorio Nacional de Oak Ridge, Tennessee, Estados Unidos. Disponible en: http://datos.bancomundial.org/indicador/EN.ATM.CO2E.PC/countries/

Benavides \& Sánchez. (2011). Indicadores ambientales para una política C-neutral en Costa Rica. El caso de la Universidad Nacional. Revista Recursos Naturales y Ambiente, 56-57, 138-144 .

Fundación Terra. (2008).Guía "X un buen clima. Calculadora de carbono. Una guía de acción contra el cambio climático". Fundación Terra y Consell Assessor per al Desenvolupament Sostenible CADS. Disponible en: http://www.terra.org/calc/index

Global Footprint Network. (2015). Huella de Carbono. 7 mayo, 2015, de GFN Extraído de: http://www. footprintnetwork.org/es/index.php/GFN/page/carbon_footprint/

Huella de Carbono. (2009). ¿Qué es la Huella de Carbono? 30 abril, 2015, de APB internet. Extraído de: http://www.huellacarbono.es/apartado/general/huella-de-carbono.html

IMN (Instituto Meteorológico Nacional, C.R.). (2014). Factores de emisión de gases de efecto invernadero ( $4^{\text {ta }}$ edic.). Autor.

IMN (Instituto Meteorológico Nacional, C.R.). (2009). Guía para la elaboración de acciones en cambio climático. Segunda Comunicación Nacional. Autor.

INTE 12-01-06:2011/Cor.2. (2013). Norma nacional para demostrar la C- neutralidad. Requisitos. Instituto de Normas Técnicas de Costa Rica Norma INTECO.

ISO 14067:2013. Requisitos y directrices para la cuantificación y comunicación de la huella de carbono de un producto (HCP) en base a las normas internacionales de evaluación del ciclo de vida.

MINAE (Ministerio de Ambiente y Energía, C.R.), IMN (Instituto Meteorológica Nacional, C.R.). (2014). Inventario nacional de gases de efecto invernadero y absorción de carbono 2010. San José, Costa Rica: Autor.

MINAET (Ministerio de Ambiente, Energía y Telecomunicaciones, C.R.), MS (Ministerio de Salud, C.R.). (2011). Guía para la elaboración de Programas de Gestión Ambiental Institucional (PGAI) en el sector público de Costa Rica. Documento de orientación para las Instituciones Públicas. San José, Costa Rica: Autor.

Programa UNA Campus Sostenible, Universidad Nacional de Costa Rica. (2014). Sistema de indicadores y base de datos. UNA Carbono Neutral-Inventario.

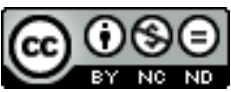

Medición de la huella de carbono de la Universidad Nacional de Costa Rica para el periodo 2012-2014. (Fabián ChavarríaSolera y otros) por Revista Uniciencia se encuentra bajo una Licencia CreativeCommons Atribución-NoComercial-SinDerivadas 3.0 Unported. 
UNICIENCIA Vol. 30, No. 2, pp. 47-62. Julio-diciembre, 2016.

ISSN Electrónico: 2215-3470

URL: www.revistas.una.ac.cr/uniciencia

DOI: http://dx.doi.org/10.15359/ru.30-2.4

Email: revistauniciencia@una.cr

\section{Anexos}

Tabla 3.

Factores de emisión de gases de efecto invernadero oficializados por el IMN para el cálculo de la huella de carbono

\begin{tabular}{|c|c|c|c|c|}
\hline \multirow{3}{*}{\multicolumn{2}{|c|}{ Aspecto }} & \multicolumn{3}{|c|}{ Factores de emisión de GEI del IMN } \\
\hline & & \multicolumn{3}{|c|}{ Año } \\
\hline & & 2012 & 2013 & 2014 \\
\hline \multicolumn{2}{|l|}{ Energía eléctrica } & $0,0824 \mathrm{~kg} \mathrm{CO} \mathrm{CO}_{2} \mathrm{e} / \mathrm{kWh}$ & $0,0771 \mathrm{~kg} \mathrm{CO} \mathrm{CO}_{2} \mathrm{e} / \mathrm{kWh}$ & $0,1300 \mathrm{~kg} \mathrm{CO} \mathrm{CO}_{2} \mathrm{e} / \mathrm{kWh}$ \\
\hline \multicolumn{2}{|l|}{ Combustible fósil } & $2,69 \mathrm{~kg} \mathrm{CO} / \mathrm{L}$ & $2,69 \mathrm{~kg} \mathrm{CO} / \mathrm{L}$ & $2,69 \mathrm{~kg} \mathrm{CO} / \mathrm{L}$ \\
\hline \multicolumn{2}{|l|}{ Gas LP } & $1,61 \mathrm{~kg} \mathrm{CO} / \mathrm{L}$ & $1,61 \mathrm{~kg} \mathrm{CO} / \mathrm{L}$ & $1,61 \mathrm{~kg} \mathrm{CO} / \mathrm{L}$ \\
\hline \multicolumn{2}{|l|}{ Residuos sólidos } & $0,0581 \mathrm{~kg} \mathrm{CH}_{4} / \mathrm{kg}$ & $0,0581 \mathrm{~kg} \mathrm{CH}_{4} / \mathrm{kg}$ & $0,0581 \mathrm{~kg} \mathrm{CH}_{4} / \mathrm{kg}$ \\
\hline \multicolumn{2}{|l|}{ Viajes aéreos } & $0,40 \mathrm{~kg}$ de $\mathrm{CO}_{2} / \mathrm{km}$ & $0,40 \mathrm{~kg}$ de $\mathrm{CO}_{2} / \mathrm{km}$ & $0,40 \mathrm{~kg}$ de $\mathrm{CO}_{2} / \mathrm{km}$ \\
\hline \multicolumn{2}{|l|}{ Aguas residuales } & $\begin{array}{l}0,876 \mathrm{~kg} \mathrm{CH}_{4} / \text { persona/ } \\
\text { año }\end{array}$ & $\begin{array}{l}0,876 \mathrm{~kg} \mathrm{CH}_{4} / \\
\text { persona/año }\end{array}$ & $\begin{array}{l}0,876 \mathrm{~kg} \mathrm{CH}_{4} / \\
\text { persona/año }\end{array}$ \\
\hline \multirow{4}{*}{$\begin{array}{l}\text { Ganado Proceso } \\
\text { digestivo }\end{array}$} & \multirow{4}{*}{$\begin{array}{l}\text { Terneros } \\
\text { Hembras en } \\
\text { crecimiento } \\
\text { Hembra adulta } \\
\text { Macho adulto }\end{array}$} & $20,00 \mathrm{~kg} \mathrm{CH}_{4} /$ cabeza & $20,00 \mathrm{~kg} \mathrm{CH}_{4} /$ cabeza & $20,00 \mathrm{~kg} \mathrm{CH}_{4} / \mathrm{cabeza}$ \\
\hline & & $48,69 \mathrm{~kg} \mathrm{CH}_{4} /$ cabeza & $48,69 \mathrm{~kg} \mathrm{CH}_{4} /$ cabeza & $48,69 \mathrm{~kg} \mathrm{CH}_{4} / \mathrm{cabeza}$ \\
\hline & & $85,00 \mathrm{~kg} \mathrm{CH}_{4} /$ cabeza & $85,00 \mathrm{~kg} \mathrm{CH}_{4} /$ cabeza & $85,00 \mathrm{~kg} \mathrm{CH}_{4} / \mathrm{cabeza}$ \\
\hline & & $111,70 \mathrm{~kg} \mathrm{CH}_{4} /$ cabeza & $111,70 \mathrm{~kg} \mathrm{CH}_{4} / \mathrm{cabeza}$ & $111,70 \mathrm{~kg} \mathrm{CH}_{4} /$ cabeza \\
\hline \multicolumn{2}{|c|}{ Ganado Manejo del estiércol } & $1 \mathrm{~kg} \mathrm{CH}_{4} /$ cabeza & $1 \mathrm{~kg} \mathrm{CH}_{4} /$ cabeza & $1 \mathrm{~kg} \mathrm{CH}_{4} /$ cabeza \\
\hline \multirow{2}{*}{ Cerdos } & Proceso digestivo & $1 \mathrm{~kg} \mathrm{CH}_{4} /$ cabeza & $1 \mathrm{~kg} \mathrm{CH}_{4} /$ cabeza & $1 \mathrm{~kg} \mathrm{CH}_{4} /$ cabeza \\
\hline & Manejo del estiércol & $1 \mathrm{~kg} \mathrm{CH}_{4} /$ cabeza & $1 \mathrm{~kg} \mathrm{CH}_{4} /$ cabeza & $1 \mathrm{~kg} \mathrm{CH}_{4} /$ cabeza \\
\hline \multirow{2}{*}{ Caballos } & Proceso digestivo & $18 \mathrm{~kg} \mathrm{CH}_{4} /$ cabeza & $18 \mathrm{~kg} \mathrm{CH}_{4} /$ cabeza & $18 \mathrm{~kg} \mathrm{CH}_{4} /$ cabeza \\
\hline & Manejo del estiércol & $1,64 \mathrm{~kg} \mathrm{CH}_{4} /$ cabeza & $1,64 \mathrm{~kg} \mathrm{CH}_{4} /$ cabeza & $1,64 \mathrm{~kg} \mathrm{CH}_{4} /$ cabeza \\
\hline \multirow{2}{*}{$\begin{array}{l}\text { Ovejas y } \\
\text { cabras }\end{array}$} & Proceso digestivo & $5 \mathrm{~kg} \mathrm{CH}_{4} /$ cabeza & $5 \mathrm{~kg} \mathrm{CH}_{4} /$ cabeza & $5 \mathrm{~kg} \mathrm{CH}_{4} /$ cabeza \\
\hline & Manejo del estiércol & $0,17 \mathrm{~kg} \mathrm{CH}_{4} /$ cabeza & $0,17 \mathrm{~kg} \mathrm{CH}_{4} /$ cabeza & $0,17 \mathrm{~kg} \mathrm{CH}_{4} /$ cabeza \\
\hline \multicolumn{2}{|l|}{ Aves de corral } & $0,02 \mathrm{~kg} \mathrm{CH}_{4} /$ cabeza & $0,02 \mathrm{~kg} \mathrm{CH}_{4} /$ cabeza & $0,02 \mathrm{~kg} \mathrm{CH}_{4} /$ cabeza \\
\hline
\end{tabular}

Fuente: $\underline{I M N}, 2014$ 